\title{
PKM $\zeta$ Restricts Dendritic Arbor Growth by Filopodial and Branch Stabilization within the Intact and Awake Developing Brain
}

\author{
Xue Feng Liu, Parisa Karimi Tari, and Kurt Haas \\ Graduate Program in Neuroscience, Brain Research Centre, Department of Cellular and Physiological Sciences, University of British Columbia, Vancouver, \\ British Columbia V6T 2B5, Canada
}

\begin{abstract}
The molecular mechanisms underlying activity-dependent neural circuit growth and plasticity during early brain development remain poorly understood. Protein kinase $\mathrm{M} \zeta(\mathrm{PKMz})$, an endogenous constitutively active kinase associated with late-phase long-term synaptic potentiation and memory in the mature brain, is expressed in the embryonic Xenopus retinotectal system with heightened levels during peak periods of dendrite growth and synaptogenesis. In vivo rapid time-lapse imaging of actively growing tectal neurons and comprehensive three-dimensional tracking of dynamic dendritic growth behavior finds that altered PKMz activity affects morphologic stabilization. Exogenous expression of PKMz within single neurons stabilizes dendritic filopodia by increasing dendritic filopodial lifetimes and decreasing filopodial additions, eliminations, and motility, whereas long-term in vivo imaging demonstrates restricted expansion of the dendritic arbor. Alternatively, blocking endogenous PKMz activity in individual growing tectal neurons with an inhibitory peptide ( $\zeta$-inhibitory peptide) destabilizes dendritic filopodia and over long periods promotes excessive arbor expansion. Furthermore, inhibiting endogenous PKMz throughout the tectum decreases colocalization of immunostained presynaptic and postsynaptic markers, SNAP-25 and PSD-95, respectively, suggesting impaired synapse maintenance. Together, these results implicate PKMz activity in restricting dendritic arborization during embryonic brain circuit development through synaptotropic stabilization of dynamic processes.
\end{abstract}

\section{Introduction}

The creation of functional neural networks during early brain development requires appropriate axonal and dendritic structural growth and formation of precise synaptic connections. Although neuronal transmission and correlated interneuronal activity are associated with synaptic plasticity underlying learning and memory in the mature brain, these factors likely influence neural circuit morphological growth during embryogenesis by directing synapse formation and maturation. The "synaptotropic model" of dendrite growth suggests that activitydependent synaptogenesis directs growth by conferring morphological stabilization to otherwise labile processes, optimizing dendritic arborization and network connections to process the specific afferent activity innervating the maturing circuit. Dendritic arbor structural growth is critical to circuit function, because dendritic surface area and length limits the number and type of synaptic contacts that can be formed, and arbor morphology influences the biophysical integration of synaptic potentials. Evidence for activity-dependent synaptotropic regulation of dendritogenesis comes from in vivo rapid time-lapse imaging of neuronal growth within the developing brain, which reveals that

Received June 16, 2009; revised Aug. 12, 2009; accepted Aug. 28, 2009.

This work was supported by operating funds from the Canadian Institutes of Health Research, the Michael Smith Foundation, the Tula Foundation, and the EJLB Foundation.

Correspondence should be addressed to Dr. Kurt Haas, Brain Research Centre, University of British Columbia, 2211 Wesbrook Mall, Rm F154, Vancouver, BC V6T 2B5, Canada. E-mail: kurt.haas@ubc.ca.

D01:10.1523/JNEUROSCI.2842-09.2009

Copyright $\odot 2009$ Society for Neuroscience $\quad$ 0270-6474/09/2912229-07\$15.00/0 glutamatergic transmission and synapse formation influences stability of highly dynamic filopodia and branches ( $\mathrm{Wu}$ et al., 1999; Sin et al., 2002; Niell et al., 2004; Haas et al., 2006).

The molecular mechanisms underlying synaptotropic dendritic arborization within developing brain circuits are poorly understood; however, mounting evidence implicates shared pathways with those underlying synaptic plasticity in the mature brain. Glutamatergic transmission and activation of CaMKII, critical components of both initiation and the early phase of long-term potentiation (LTP) at mature synapses, have been shown to regulate dendritic arborization in developing central neurons in vivo (Rajan and Cline, 1998; Wu and Cline, 1998; Rajan et al., 1999; Haas et al., 2006). Here, we investigate whether mechanisms underlying late-phase LTP in the mature brain are also involved in developmental morphologic plasticity. Protein kinase $M \zeta$ $(\mathrm{PKMz})$, a recently discovered constitutively active isoform of protein kinase $\mathrm{C}$ (PKC), has been implicated in late-phase hippocampal LTP, as well as learning and memory in Drosophila and rodents (Jiang et al., 1994; Drier et al., 2002; Ling et al., 2002; Pastalkova et al., 2006). PKMz has high homology to the catalytic domain of PKC $\zeta(\mathrm{PKCz})$, yet lacks the PKC autoinhibitory domain and thereby exhibits persistent kinase activity (Sacktor et al., 1993). Intracellular introduction of PKMz to hippocampal pyramidal neurons in mature brain slices potentiates synaptic transmission and occludes LTP induction. Inhibiting endogenous PKMz activity with the specific $\zeta$-inhibitory peptide (ZIP) peptide inhibitor abolishes late-phase LTP maintenance but not LTP induction or short-term potentiation (Hrabetova and Sack- 


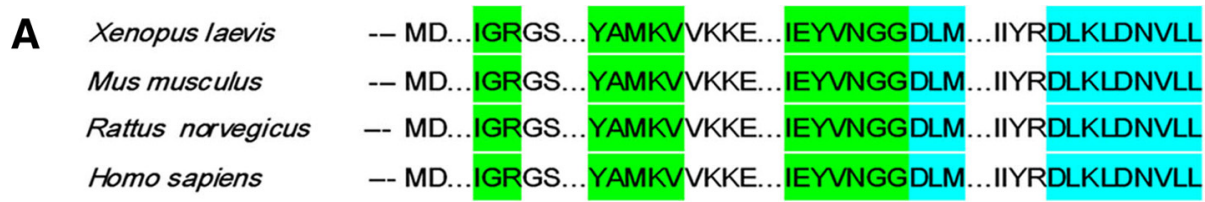
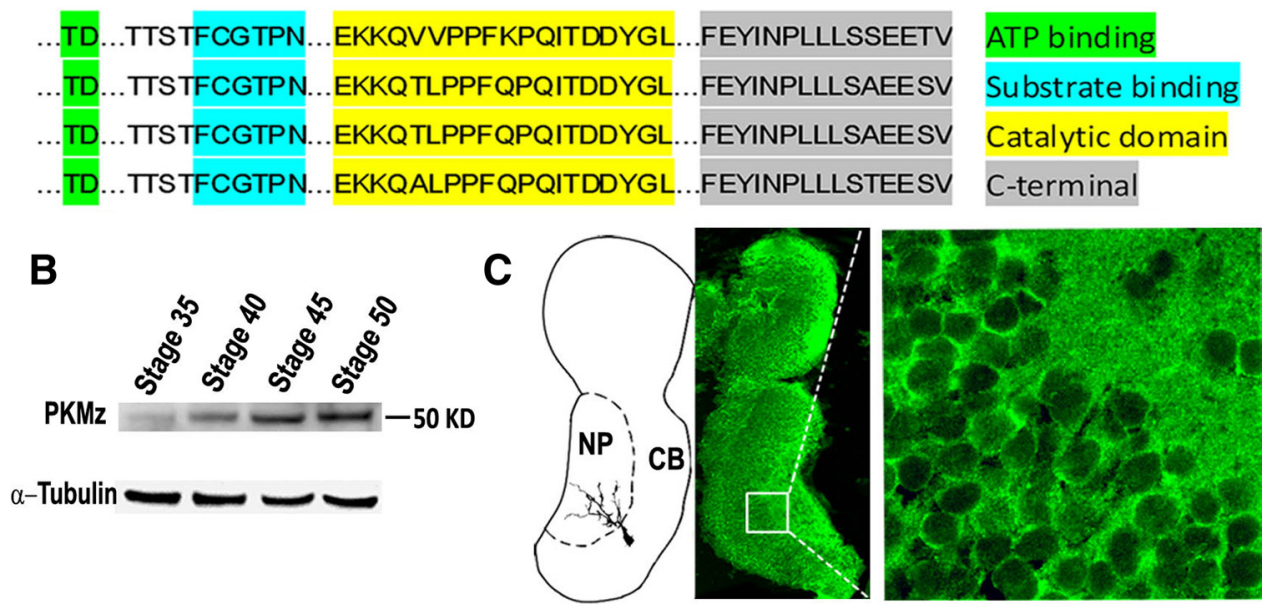

Figure 1. Characterization of Xenopus PKMz. A, Alignment of predicted PKMz amino acid sequences from Xenopus laevis and other species. Regions corresponding to postulated major motifs are highlighted. $\boldsymbol{B}$, Western blot using an antibody raised against the catalytic domain of rat PKMz identifies a 50 kDa protein from Xenopus brain extracts, demonstrates a progressive developmental increase in expression. C, Schematic drawing of tadpole brain (left) and horizontal section stained with PKMz antibody demonstrating enriched immunoreactivity in the neuropil region (NP) and the membrane portion of cell body (CB) region of the tectum.

tor, 1996; Osten et al., 1996; Ling et al., 2002). Here, we test whether PKMz mediates morphological plasticity in early brain development by controlling expression and activity of PKMz in single neurons within the retinotectal system of the intact albino Xenopus laevis tadpole brain. We use rapid and long-interval in vivo two-photon time-lapse imaging combined with comprehensive three-dimensional (3D) quantification involving tracking of all dendritic filopodia and branches. Results demonstrate bidirectional regulation of dendritic stabilization by $\mathrm{PKMz}$ activity resulting in altered arbor expansion during periods of dynamic dendritogenesis.

\section{Materials and Methods}

Animals. Freely swimming stage 47 albino Xenopus laevis tadpoles (Nieuwkoop and Faber, 1994) were reared in $10 \%$ Steinberg's solution [1X Steinberg's: $10 \mathrm{~mm}$ HEPES, $60 \mathrm{~mm} \mathrm{NaCl}, 0.67 \mathrm{~mm} \mathrm{KCl}, 0.34 \mathrm{~mm}$ $\left.\mathrm{Ca}\left(\mathrm{NO}_{3}\right)_{2}, 0.83 \mathrm{~mm} \mathrm{MgSO}_{4}, \mathrm{pH} 7.4\right]$ and housed at $22^{\circ} \mathrm{C}$ on a $12 \mathrm{~h}$ light/dark cycle. Experiments were conducted at room temperature $\left(22^{\circ} \mathrm{C}\right)$. All experimental procedures were conducted according to the guidelines of the Canadian Council on Animal Care and approved by the Animal Care Committee of the University of British Columbia's Faculty of Medicine.

DNA constructs. Xenopus PKMz was cloned from brain cDNA using reverse transcriptase-PCR and amplified using primers (upstream: 5'-GGCAAGGCTACAAGTGTATCAACTGC-3', downstream: 5'-GCTATAGAGAGATCAGCTTATACGGTCTCC- $3^{\prime}$ ) based on the sequences flanking the coding region of the Xenopus laevis PKMz cDNA (GI: 1220553), and the PCR product was inserted into a pCDNA expression vector. Green fluorescent protein (GFP) fusions of Xenopus and mouse forms of PKMz were constructed and used for overexpression studies (mouse PKMz was a gift from Dr. Jerry Yin, University of Wisconsin, Madison, WI). Mouse and Xenopus laevis sequences share $87 \%$ homology and have conserved ATP and substrate binding motifs. The kinase activity of fusion proteins was verified by using a $\mathrm{PKCz}$-specific kinase assay. No significant differences were found between the effects of expression of the Xenopus or mouse PKMz on dendrite arborization, so data from these groups were combined.
Single-cell electroporation for transfection of individual neurons in vivo. Individual immature neurons within the optic tectum of the intact tadpole brain were fluorescently labeled using single-cell electroporation (SCE) (Haas et al., 2001; Hewapathirane et al., 2008). Tadpoles were briefly anesthetized with $0.02 \%$ 3-aminobenzoic acid ethyl ester (MS222). Under visual guidance using an upright stereomicroscope, a sharp glass pipette (tip diameter, $\sim 0.6 \mu \mathrm{m}$ ) loaded with a solution of plasmid DNA $(1-2 \mu \mathrm{g} / \mu \mathrm{l})$ was inserted into the optic tectum with the tip opposed to newly differentiated neurons in the tectal proliferative zone. An Axoporator 800A (Molecular Devices) was used to deliver electric pulses. Stimulus parameters were as follows: pulse intensity, $1.5 \mu \mathrm{A}$; pulse duration, $1 \mathrm{~ms}$; pulse frequency, $300 \mathrm{~Hz}$; train duration, $300 \mathrm{~ms}$.

PKMz inhibitor peptide application. Tadpoles were anesthetized with $0.02 \% \mathrm{MS} 222$, and cell-permeable myristoylated-PKMz inhibitor peptide (mZIP) (QCB) dissolved in PBS at $0.5 \mathrm{nmol} / \mu \mathrm{l}$ and a total volume of $200 \mathrm{~nL}$ was slowly perfused into tadpole tectum using a fine glass pipette attached to a Picospritzer (General Valve). PBS was used as vehicle control. Alternatively, targeted inhibition of PKMz solely in individual neurons being imaged was achieved by SCE of ZIP peptide along with Alexa Fluor 488, 3000MW (Invitrogen) to visualize neuron morphology. An intrapipette concentration of ZIP peptide of $5 \mathrm{nmol} / \mu \mathrm{l}$ and $3 \mathrm{~mm}$ for Alexa Fluor 488 was used. Delivery stimulus parameters for SCE of peptide and dye were as follows: pulse intensity, $2 \mu \mathrm{A}$; pulse duration, $700 \mu \mathrm{s}$; pulse frequency, $700 \mathrm{~Hz}$; train duration, $20 \mathrm{~ms}$. In some experiments, TAMRA dye-labeled ZIP peptide (Pepmetric) was used to determine duration of peptide retention within neurons over days. Loading of TAMRA-labeled peptide was confirmed by fluorescence and confocal microscopy, and coelectroporation with Alexa Fluor 488 yielded $100 \%$ coloading. No significant differences were found between the effects of labeled or non-labeled ZIP on dendrite arborization, so data from these groups were combined.

Immunoblotting. Tadpoles were anesthetized with $0.02 \%$ MS222, killed, and brains were dissected and homogenized in lysis buffer $(50 \mathrm{~mm}$ Tris- $\mathrm{HCl}, 1 \times$ Roche protease inhibitor mixture, $1 \%$ Triton $\mathrm{X}-100, \mathrm{pH}$ 7.4). After centrifuge separation at $15000 \mathrm{~g}$ for $10 \mathrm{~min}$ at $4^{\circ} \mathrm{C}$, the supernatant was denatured, separated on a $4-12 \%$ Bis-Tris acrylamide gel (Invitrogen), and transferred to a nitrocellulose membrane. The blots 

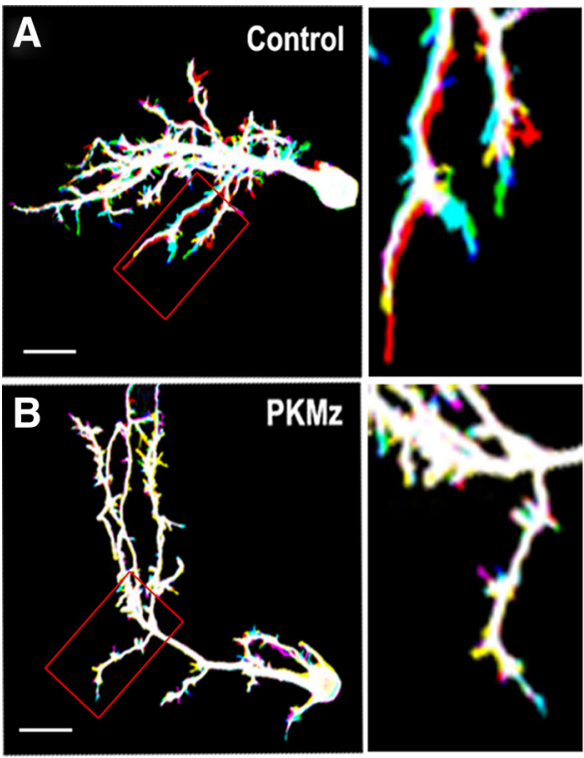

C

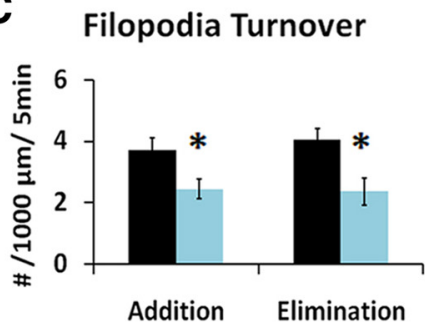

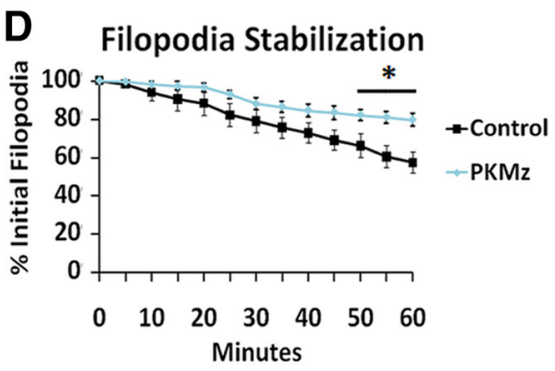

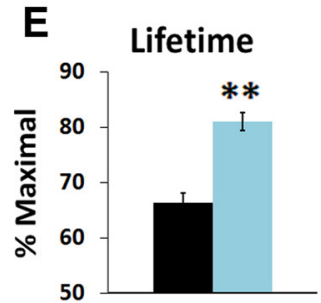

$\mathbf{F}$

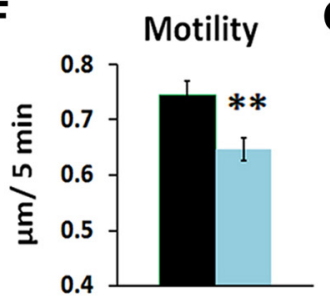

G Dynamic Range

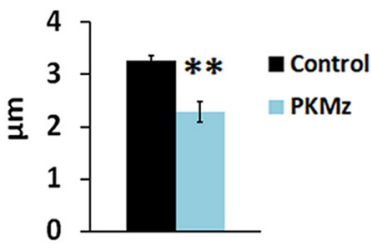

H
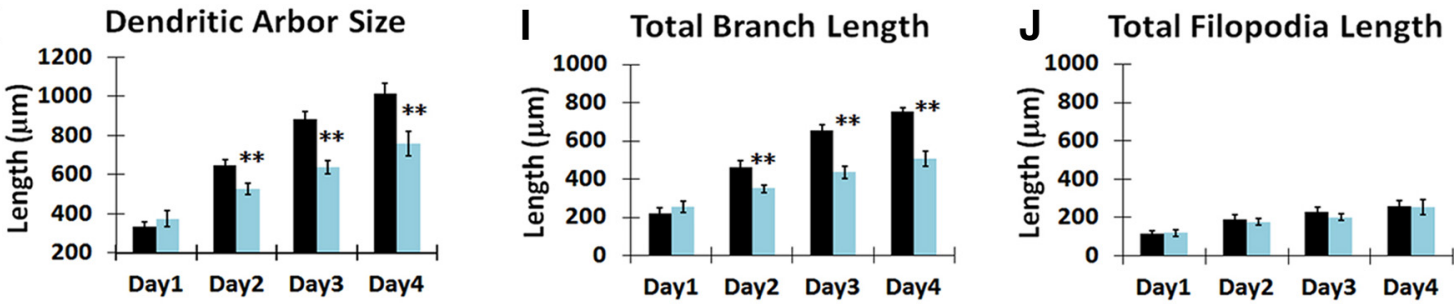

K

Sholl Analysis
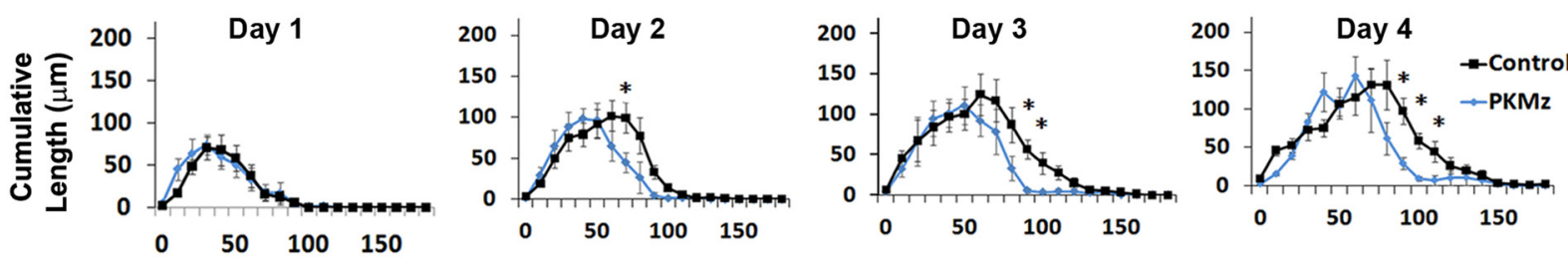

Distance from Soma $(\mu \mathrm{m})$

Figure 2. PKMz overexpression stabilizes dendritic filopodia and restricts arbor extension. $A, B$, Superimposed images of six successive time points (each a different color, with overlap $=$ white) at 10 min imaging intervals of a neuron expressing exogenous PKMz $(\boldsymbol{A})$ and a control neuron $(\boldsymbol{B})$. Area within the boxed region is enlarged on the right. Scale bars, $20 \mu \mathrm{m}$. $\boldsymbol{C}-\boldsymbol{G}, \mathrm{Morphometric}$ analysis of dendritic filopodial dynamics of PKMz neurons ( $N=6$ cells; $n=269$ filopodia) and control neurons ( $N=7$ cells; $n=255$ filopodia). PKMz overexpression significantly decreases the rates of filopodial addition and elimination $(\boldsymbol{C})$; increases the fraction of filopodia that persist for $1 \mathrm{~h}(\boldsymbol{D})$ and mean filopodial lifetime $(\boldsymbol{E})$; decreases filopodial motility $(\boldsymbol{F})$ and dynamic range $(\boldsymbol{G})$. $\boldsymbol{H}-\boldsymbol{K}, 3 \mathrm{D}$ morphometric analysis of tectal neurons expressing GFP alone (control, $n=12)$ and GFP with PKMz $(n=10) 1-4 \mathrm{~d}$ after electroporation. PKMz overexpression restricts daily addition of dendritic arbor size $(\boldsymbol{H})$, attributable to decreased extension of branches $(\boldsymbol{I})$ but not filopodia $(\boldsymbol{J}) . \boldsymbol{K}, 3 \mathrm{D}$ Sholl analysis of dendritic arbor complexity $1-4 \mathrm{~d}$ after transfection. ${ }^{*} p<0.05$; ${ }^{* *} p<0.01$.

were then probed with a PKMz antibody (a gift from Dr. T. C. Sacktor, SUNY Downstate Medical Center, Brooklyn, NY).

Immunohistochemistry. For immunostaining of brain sections, tadpoles were fixed in $4 \%$ paraformaldehyde in $\mathrm{PBS}$ for $2 \mathrm{~h}$ at room temperature followed by cryoprotection in a solution of $30 \%$ sucrose at $4{ }^{\circ} \mathrm{C}$ overnight and sliced at $20 \mu \mathrm{m}$ (horizontal cryosections). The sections were blocked in $5 \%$ normal goat serum for $1 \mathrm{~h}$ and incubated in primary antibodies at $4^{\circ} \mathrm{C}$ overnight. After washing with PBS buffer, sections were incubated in Alexa Fluor-conjugated secondary antibodies (Invitrogen) for $1 \mathrm{~h}$ at room temperature, mounted, and imaged with an Olympus FV-1000 confocal microscope. The primary antibodies and dilutions used were: PKMz antibody (rabbit polyclonal, 1:100), SNAP-25 (rabbit polyclonal, 1:400; Stressgen), and PSD-95 (mouse IgG, 1:200; Millipore Bioscience Research Reagents). For secondary antibodies, we used Alexa 568-conjugated goat anti-rabbit antibody and Alexa 633-conjugated goat anti-mouse antibody (1:200). For measurements of immunostained PSD-95 and SNAP-25 puncta density, all puncta larger than $0.2 \mu \mathrm{m}^{2}$ were included. The cutoff size was selected based on the size distribution of "false-positive" puncta on images from brain sections incubated with only secondary antibodies. The density of total PSD-95 and SNAP-25 puncta, as well as colocalized puncta, was determined using National Institutes of Health ImageJ software. Colocalization probability was calculated by multiplying the percentage of colocalized puncta in each channel. Results from mZIP sections were normalized to control sections on the same staining slide and tested for significance with unpaired Student's $t$ tests. Colocalization was dramatically decreased by rotating either PSD-95 or SNAP- 25 images by $90^{\circ}$ to each other $(43.2 \pm 2.6 \%$ of original value).

In vivo time-lapse two-photon imaging of dendritogenesis. Images of neurons were captured using a custom-built two-photon microscope consisting of a modified Olympus Fluoview $300 \mathrm{~V}$ confocal coupled to a Chameleon Ti:Sapphire laser (Coherent). An Olympus LUMPlanFl/IR $60 \times$, water-immersion objective (1.1 NA) was used, with a $z$-axis step size of $1.5 \mu \mathrm{m}$. To capture rapid dendritic filopodial dynamics, unanesthetized tadpoles were immobilized for imaging using the reversible paralytic panacurarium dibromide ( $3 \mathrm{~mm}$; Tocris) and embedded 

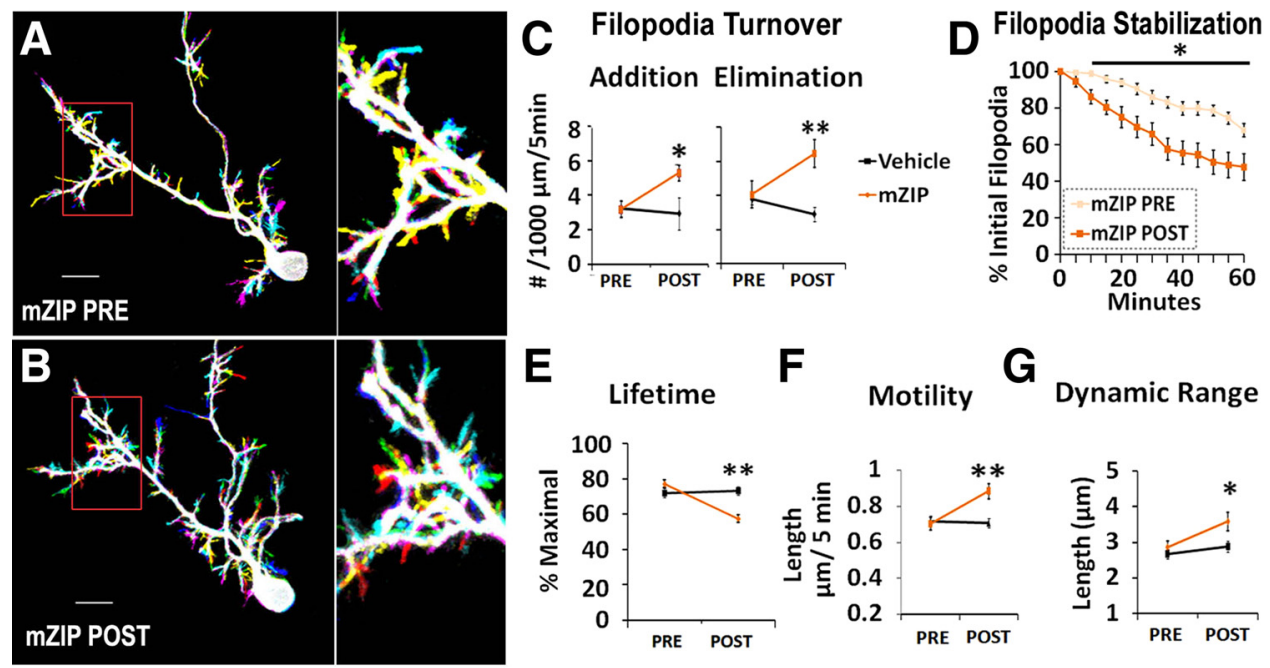

Fotility

G
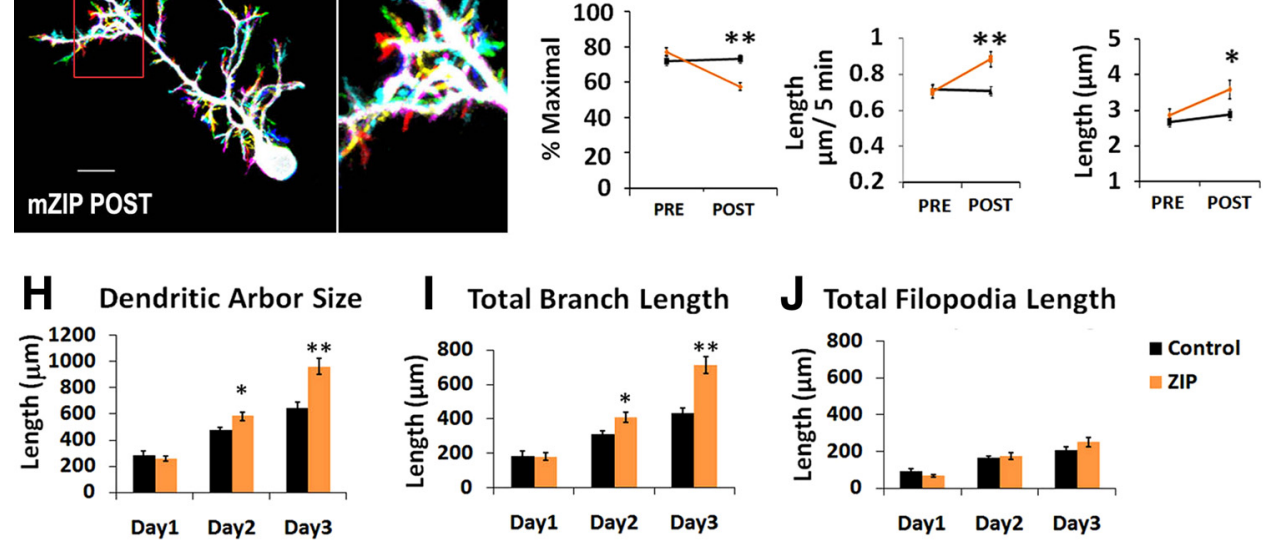

J Total Filopodia Length

$\mathbf{K}$

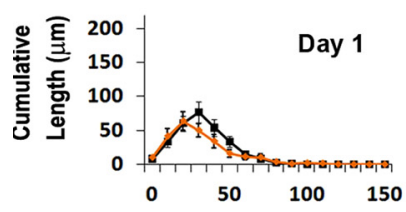

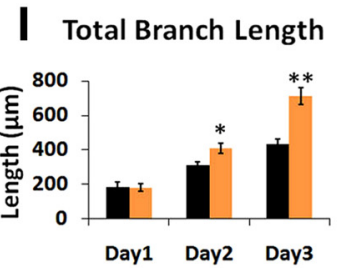

Sholl Analysis
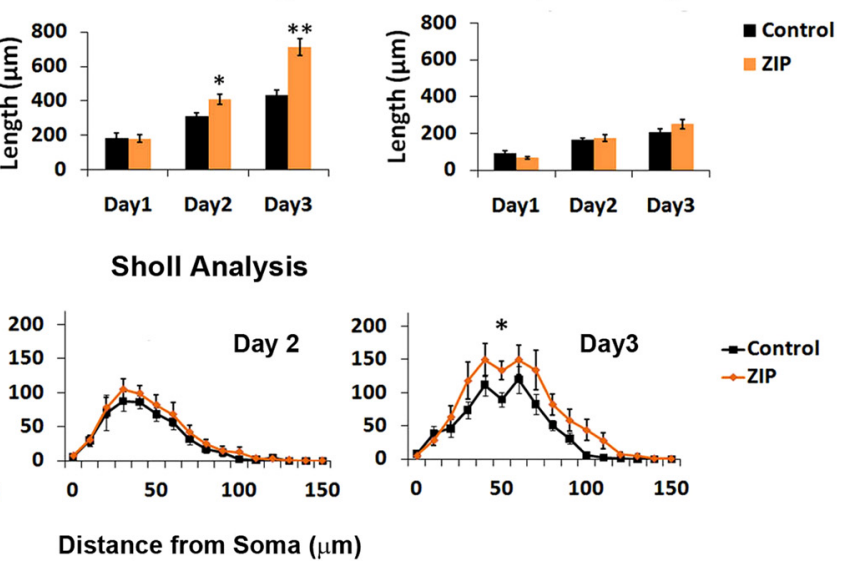

Distance from Soma $(\mu \mathrm{m})$

Figure 3. ZIP destabilizes dendritic filopodia and promotes arbor extension. $A, B$, Overlay of six successive images at $10 \mathrm{~min}$ of a GFP neuron before $(\boldsymbol{A})$ and $4 \mathrm{~h}$ after $\mathrm{mZIP}$ treatment $(\boldsymbol{B})$. White portions of the arbor are stable, and colored portions are dynamic over $1 \mathrm{~h}$ imaging period. The area within the boxed region is enlarged on the right. Scale bar, $20 \mu \mathrm{m}$. $C-G$, Morphometric analysis of dendritic filopodial dynamics of neurons before and after mZIP delivery to tectum ( $N=5$ cells $; n=213$ filopodia for PRE and 215 filopodia for POST) and vehicle $(N=5$ cells; $n=221$ filopodia for PRE and 210 filopodia for POST). mZIP treatment significantly increases the rates of filopodial addition and elimination (C), decreases the proportion of stabilized pre-existing filopodia (D) and mean filopodial lifetime $(\boldsymbol{E})$; increases the filopodial motility $(\boldsymbol{F})$ and dynamic range $(\boldsymbol{G}) . \boldsymbol{H}-\boldsymbol{K}, 3 \mathrm{D}$ morphometric analysis of tectal neurons labeled with Alexa Fluor 488 dye alone (control, $n=12$ ) and with ZIP $(n=13) 1-3$ d after electroporation. ZIP increases total dendritic arbor size $(\boldsymbol{H})$ and branch length $(\boldsymbol{I})$ but not total filopodial length $(\boldsymbol{J})$. $\boldsymbol{K}$, 3D Sholl analysis of dendritic arbor complexity $1-3$ d after electroporation. ${ }^{*} p<0.05 ;{ }^{* *} p<0.01$.

under a thin layer of agarose $(0.8 \%)$ in a chamber continuously perfused with oxygenated $10 \%$ Steinberg's solution. 3D stacks of images through entire dendritic arbors were captured every 5 min over $1 \mathrm{~h}$. Long-term dendrite growth was imaged with $24 \mathrm{~h}$ intervals. Single stacks of images through the entire dendritic arbor were captured by briefly anesthetizing tadpoles with $0.02 \% \mathrm{MS} 222$. After imaging, tadpoles were returned to rearing solution where they rapidly recovered from anesthesia.

Morphometric analysis of dendritic growth. Three-dimensional skeletonized reconstructions of entire dendritic arbors were constructed using Neurolucida software (MicroBrightfield) and used to analyze total dendritic branch length, branch tip number, and 3D Sholl analysis. All morphometric data analysis, except for Sholl analysis, was tested for significance with unpaired Student's $t$ tests. Results from Sholl analysis were tested with one-way ANOVA tests, followed by Turkey post hoc analysis. Rapid dendritic filopodial and branch growth dynamics were analyzed using custom-written software using the IGOR platform (based on a program created by Dr. Jamie Boyd, University of British Columbia, Vancouver, BC, Canada). All filopodia and branches were identified and tracked across multiple time point image stacks.

\section{Results}

Endogenous expression of PKMz in Xenopus tadpole brain A PKMz mRNA previously identified in Xenopus laevis (GI: 1220553 ) was used as the basis for PCR primers to clone PMKz and confirm expression in tadpole brain. Putative translation of this mRNA generates a protein containing 401 aa with $87 \%$ homology to rat PKMz (Hernandez et al., 2003) and conserved ATP and substrate-binding motifs (Fig. 1A). Using an antibody raised against the catalytic domain of rat $\mathrm{PKCz}$ and immunoblots, we detected $\mathrm{PKMz}$ in tadpole brain lysates as a $50 \mathrm{kDa}$ protein, consistent with its putative translation product. Western blot analysis found developmental regulation of $\mathrm{PKMz}$ expression in tadpole brain, with levels increasing significantly from stage 35 to stage 50 (Fig. $1 \mathrm{~B}$ ). This period spans initial innervation and subsequent activity-dependent refinement of afferent axonal input from retinal ganglion cells (RGCs) and tectal neuron dendritogenesis (Grant et al., 1980). Immunohistochemistry staining of tadpole brain cryosections 
A

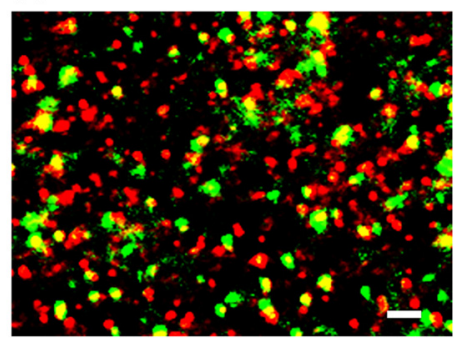

Control

\section{B}

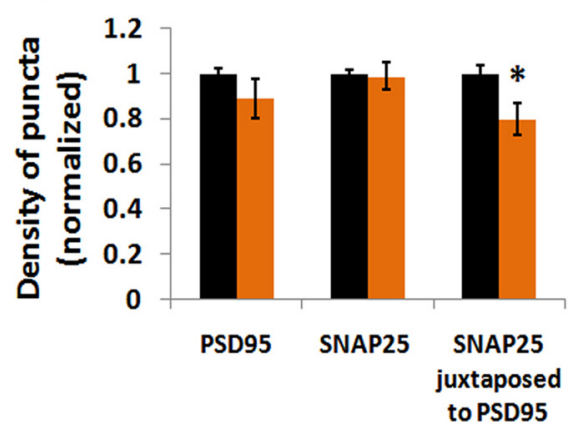

SNAP25 PSD95

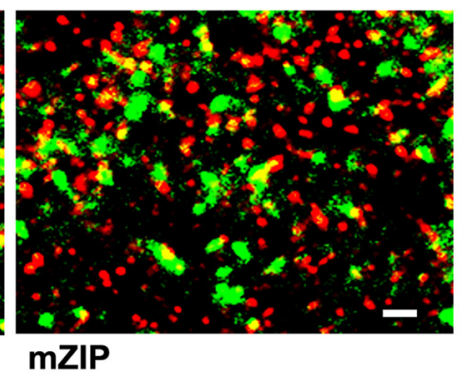

C

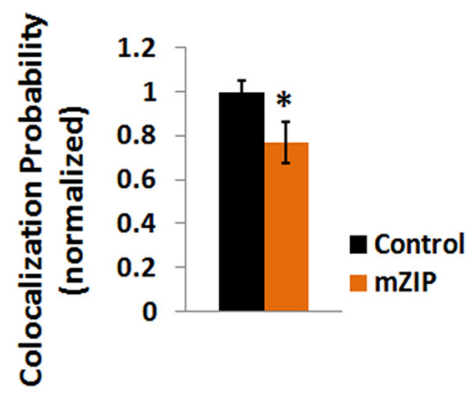

Figure 4. $\mathrm{mZIP}$ impairs maintenance of retinotectal synapses in vivo. $\boldsymbol{A}$, Representative immunostaining of the tectal neuropil from a horizontal brain section with antibodies against SNAP-25 (red) and PSD-95 (green) $4 \mathrm{~h}$ after tectal infusion of vehicle (control) and mZIP. Yellow puncta represent the overlap of SNAP-25 and PSD-95 puncta. Scale bars, $1 \mu \mathrm{m}$. $\boldsymbol{B}, \boldsymbol{C}$, Quantitative analysis of the density of SNAP-25 puncta, PSD-95 puncta, and juxtaposed puncta (B), as well as colocalization probability $(\boldsymbol{C})$, normalized by the average value of the corresponding controls $(n=5$ tadpoles for each condition; * $p<0.05$ ).

found PKMz immunoreactivity enriched in tectal neuropil where innervating RGC axons synapse with tectal neuron dendrites (Fig. 1C). Therefore, the endogenous expression of $\mathrm{PKMz}$ in Xenopus tadpole brain demonstrates a spatial-temporal pattern consistent with a role in synaptic and morphologic plasticity.

\section{PKMz overexpression stabilizes dendritic filopodial growth dynamics}

In developing neurons, dendritic filopodia are highly dynamic actin-rich protrusions typically $<10 \mu \mathrm{m}$ in length that emerge from the dendritic shaft and are precursors of longer branches (Portera-Cailliau et al., 2003; Niell et al., 2004). The majority of these processes retract within tens of minutes after initial extension, but a small percentage become stabilized and elongate to form new persistent dendritic branches. To comprehensively characterize filopodial growth dynamics, we used rapid in vivo time-lapse imaging followed by tracking and measuring all processes at $5 \mathrm{~min}$ intervals for $1 \mathrm{~h}$. Measures allowed precise quantification of dendritic filopodia and branch motility, addition and elimination rates, and lifetimes. Results from rapid time-lapse imaging and analysis of growing tectal neurons transfected to express exogenous PKMz found a marked increase in filopodial stabilization compared with controls (Fig. $2 A, B$ ). PKMz overexpression induced a significant reduction in rates of filopodial addition and elimination (Fig. 2C), resulting in a decrease in overall turnover rate. Accordingly, the percentage of stable filopodia existing over the entire $1 \mathrm{~h}$ imaging period, and average filopodial lifetime was significantly higher in PKMz neurons than controls (Fig. 2D,E). Furthermore, PKMz overexpression reduced both the absolute filopodial motility, represented by

average length change per $5 \mathrm{~min}$, and the filopodial dynamic range, the maximal change in length during the imaging period (Fig. $2 F, G$ ).

\section{PKMz overexpression restricts dendritic arbor expansion} Effects of altered PKMz activity on longterm dendrite arborization were examined by imaging-transfected developing tectal neurons at $24 \mathrm{~h}$ intervals over several days. Dendrite morphologies of neurons overexpressing PKMz did not differ from controls at the initial imaging time point $12 \mathrm{~h}$ after electroporation, but their growth patterns diverged in subsequent days (Fig. 2). Over 4 d of imaging, dendritic arbors of control neurons increased in size and complexity, whereas neurons expressing exogenous PKMz exhibited restricted arbor expansion. Increased $\mathrm{PKMz}$ activity produced a reduction in daily addition of total dendrite arbor length (Fig. $2 \mathrm{H}, I$ ) and reduced arbor complexity (Fig. $2 K$ ), without significantly altering dendrite branch tip number (data not shown) or total length or number of filopodia (Fig. $2 \mathrm{~J}$ ). Results demonstrate a persistent restriction of branch extension.

\section{Inhibition of endogenous PKMz destabilizes dendritic filopodia}

To examine the role of endogenous PKMz activity in regulating dynamic dendritic growth, we imaged rapid growth behavior during a $1 \mathrm{~h}$ baseline, followed by intratectal infusion of the selective PKMz inhibitor mZIP. After $4 \mathrm{~h}$ incubation, a second $1 \mathrm{~h}$ rapid-imaging session was conducted. Within-cell comparisons found significant destabilization of dendritic filopodia induced by PKMz inhibition (Fig. $3 A, B$ ). Intratectal mZIP infusion destabilized dendritic filopodia, clear from a significant increased turnover rates of dendritic filopodia attributable to increased rates of both filopodial addition and elimination (Fig. 3C), reduction in the maintenance of pre-existing filopodia (Fig. 3D), and average filopodial lifetime (Fig. $3 E$ ). Furthermore, the absolute motility and dynamic range of filopodial growth were significantly increased (Fig. $3 F, G$ ).

\section{Long-term inhibition of endogenous PKMz promotes dendritic arbor expansion}

The role of endogenous PKMz activity in the long-term growth patterns of dendritic arbors was examined by delivering the ZIP PKMz inhibitor peptide along with Alexa Fluor dye into individual developing tectal neurons using SCE. Labeled neurons were imaged $6 \mathrm{~h}$ after electroporation to capture initial baseline images before significant effects of ZIP on growth and to match control and ZIP neurons based on initial arbor size. Daily dendritic arbor growth was imaged over $3 \mathrm{~d}$, whereas fluorescently tagged peptide (TAMRA dye-labeled ZIP) could be reliably detected within neurons after SCE delivery during this period. Inhibition of PKMz by intracellular application of ZIP promoted increased dendrite outgrowth compared with dye-labeled controls, represented by significantly larger total dendritic branch length (Fig. $3 H$ ). In contrast, dendritic branch tip number was not significantly af- 
fected by ZIP application (data not shown). 3D Sholl analysis found that $\mathrm{PKMz}$ inhibition increases arbor expansion as well as complexity (Fig. $3 \mathrm{~K}$ ). The primary effect of inhibition of endogenous PKMz by ZIP was an enhanced extension of branches, with no effects on the total filopodial length or number (Fig. $3 I, J$ ).

\section{PKMz inhibition impairs synapse maintenance in vivo}

Given the role of PKMz in synapse plasticity in the adult brain and the importance of synaptogenesis in filopodial stabilization during dendritogenesis, we examined the effects of PKMz on synapse density in tadpole tectum. We used immunostaining of tadpole brain cryosections for the presynaptic and postsynaptic markers SNAP-25 and PSD-95. PKMz inhibition by intratectal infusion of mZIP significantly reduced tectal synapse density after $4 \mathrm{~h}$, evident from decreased density of PSD-95 puncta juxtaposed to SNAP-25 puncta and colocalization probability. No significant changes were found in the densities of either puncta (Fig. 4). These results demonstrate that inhibition of PKMz during active periods of developmental synaptogenesis impairs maintenance of excitatory synapses.

\section{Discussion}

The synaptotropic model of dendritogenesis postulates that synapses stabilize growing dendritic processes, thereby preventing retraction, yet allowing further growth and branching from synaptic sites (Vaughn, 1989; Niell et al., 2004; Cline and Haas, 2008). However, this model does not explain the progressive decline in dendritic growth and structural plasticity as neurons mature, characterized by reduced growth, motility, and additions of dendritic filopodia and branches (Wu et al., 1999). A possible explanation is that synaptotropic dendritogenesis is influenced by the maturational state of constituent glutamatergic synapses, thereby linking growth with the functional maturation of neurons ( $\mathrm{Wu}$ et al., 1996). In this framework, nascent immature synapses forming on new filopodia act to prevent retraction while permitting further growth. Mature synapses, in contrast, would restrict continued growth by conferring further structural stabilization. Here, we identify PKMz, a naturally expressed constitutively active kinase mediating late-phase synaptic LTP in the mature brain (Ling et al., 2002), as a potential regulator of synapse maturation state-dependent synaptotropic dendrite growth.

Increasing or decreasing levels of PKMz activity in individual actively growing brain neurons produces a similar shift in dendritic growth plasticity. Increasing $\mathrm{PKMz}$ activity in immature neurons dramatically reduces dendritic morphological plasticity, resulting in stunted arbors demonstrating precocious stabilization similar to mature neurons. PKMz's stabilizing effects on growing dendrites are likely mediated through synapse maturation rather than a direct effect on the cytoskeleton, since we found no effects on the motility of new filopodia within the first $30 \mathrm{~min}$ of extension, a period before detectable synapse formation (Niell et al., 2004) (data not shown). Inhibiting endogenous PKMz activity with ZIP, shown to prevent late-phase synaptic potentiation in the mature brain (Ling et al., 2002), and here to reduce synapse density in the Xenopus tectum, destabilizes growing dendrites with increased dendritic motility and turnover.

The identification of a role for PKMz in dendritogensis adds to a growing list of molecular pathways shared between developmental neuronal morphogenesis and synaptic plasticity in the mature brain. Glutamateric transmission, critical to initiation of mature synaptic plasticity, promotes dendrite growth (Sin et al., 2002), whereas inhibition of NMDA or AMPA receptor transmission destabilizes filopodia and inhibits growth (Rajan and
Cline, 1998; Sin et al., 2002; Haas et al., 2006). At mature synapses, calcium entry through NMDA receptors activates CaMKII, a critical component of early phase LTP. Exogenous expression of a constitutively active form of CaMKII in immature Xenopus tectal neurons both promotes maturation of retinotectal glutamatergic synapses (Wu et al., 1996) and restricts dendritic arbor plasticity similar to increasing $\mathrm{PKMz}$ activity ( $\mathrm{Wu}$ and Cline, 1998). Inhibition of endogenous CaMKII activity destabilizes growing dendrites. Because PKMz expression is regulated by activity of CaMKII during induction of LTP in the mature hippocampus (Kelly et al., 2007), these kinases may act in concert during development to confer early and late-phase synaptic maturation and associated morphologic stabilization. These findings suggests that synapse maturation during brain circuit development which underlies morphological stabilization involves molecular mechanisms common to synaptic plasticity associated with learning and memory in the mature brain.

Manipulations predicted to shift synaptic plasticity in growing neurons in vivo, however, do not produce arbor morphologies predicted from a simple interpretation of the synaptotropic model, where enhancing synapse formation and maturation would be expected to promote growth, whereas the inhibition of synaptic strengthening would limit growth. Rather, enhanced PKMz or CaMKII activity, expected to lower a neuron's threshold for synapse maturation, results in stunted, nonmotile arbors, whereas inhibiting these plasticity-associated kinases produces unrestricted arbor elongation. Thus, synapse maturation during dendritogenesis appears to function not only to stabilize nascent processes to prevent retraction but also to restrict excessive arborization and may underlie the loss of dendrite morphological plasticity with neuronal maturation.

\section{References}

Cline H, Haas K (2008) The regulation of dendritic arbor development and plasticity by glutamatergic synaptic input: a review of the synaptotrophic hypothesis. J Physiol 586:1509-1517.

Drier EA, Tello MK, Cowan M, Wu P, Blace N, Sacktor TC, Yin JC (2002) Memory enhancement and formation by atypical PKM activity in Drosophila melanogaster. Nat Neurosci 5:316-324.

Grant P, Rubin E, Cima C (1980) Ontogeny of the retina and optic nerve in Xenopus laevis. I. Stages in the early development of the retina. J Comp Neurol 189:593-613.

Haas K, Sin WC, Javaherian A, Li Z, Cline HT (2001) Single-cell electroporation for gene transfer in vivo. Neuron 29:583-591.

Haas K, Li J, Cline HT (2006) AMPA receptors regulate experiencedependent dendritic arbor growth in vivo. Proc Natl Acad Sci U S A 103:12127-12131.

Hernandez AI, Blace N, Crary JF, Serrano PA, Leitges M, Libien JM, Weinstein G, Tcherapanov A, Sacktor TC (2003) Protein kinase M zeta synthesis from a brain mRNA encoding an independent protein kinase $\mathrm{C}$ zeta catalytic domain. Implications for the molecular mechanism of memory. J Biol Chem 278:40305-40316.

Hewapathirane DS, Dunfield D, Yen W, Chen S, Haas K (2008) In vivo imaging of seizure activity in a novel developmental seizure model. Exp Neurol 211:480-488.

Hrabetova S, Sacktor TC (1996) Bidirectional regulation of protein kinase $\mathrm{M} \zeta$ in the maintenance of long-term potentiation and long-term depression. J Neurosci 16:5324-5333.

Jiang X, Naik MU, Hrabe J, Sacktor TC (1994) Developmental expression of the protein kinase $\mathrm{C}$ family in rat hippocampus. Brain Res Dev Brain Res 78:291-295.

Kelly MT, Crary JF, Sacktor TC (2007) Regulation of protein kinase M $\zeta$ synthesis by multiple kinases in long-term potentiation. J Neurosci 27:3439-3444.

Ling DS, Benardo LS, Serrano PA, Blace N, Kelly MT, Crary JF, Sacktor TC (2002) Protein kinase Mzeta is necessary and sufficient for LTP maintenance. Nat Neurosci 5:295-296. 
Niell CM, Meyer MP, Smith SJ (2004) In vivo imaging of synapse formation on a growing dendritic arbor. Nat Neurosci 7:254-260.

Nieuwkoop PD, Faber J (1994) Normal table of Xenopus laevis. Amsterdam: Elsevier.

Osten P, Valsamis L, Harris A, Sacktor TC (1996) Protein synthesisdependent formation of protein kinase $\mathrm{M} \zeta$ in long-term potentiation. J Neurosci 16:2444-2451.

Pastalkova E, Serrano P, Pinkhasova D, Wallace E, Fenton AA, Sacktor TC (2006) Storage of spatial information by the maintenance mechanism of LTP. Science 313:1141-1144.

Portera-Cailliau C, Pan DT, Yuste R (2003) Activity-regulated dynamic behavior of early dendritic protrusions: evidence for different types of dendritic filopodia. J Neurosci 23:7129-7142.

Rajan I, Cline HT (1998) Glutamate receptor activity is required for normal development of tectal cell dendrites in vivo. J Neurosci 18:78367846.

Rajan I, Witte S, Cline HT (1999) NMDA receptor activity stabilizes presyn- aptic retinotectal axons and postsynaptic optic tectal cell dendrites in vivo. J Neurobiol 38:357-368.

Sacktor TC, Osten P, Valsamis H, Jiang X, Naik MU, Sublette E (1993) Persistent activation of the zeta isoform of protein kinase $\mathrm{C}$ in the maintenance of long-term potentiation. Proc Natl Acad Sci U S A 90:8342-8346.

Sin WC, Haas K, Ruthazer ES, Cline HT (2002) Dendrite growth increased by visual activity requires NMDA receptor and Rho GTPases. Nature 419:475-480.

Vaughn JE (1989) Fine structure of synaptogenesis in the vertebrate central nervous system. Synapse 3:255-285.

Wu G, Malinow R, Cline HT (1996) Maturation of a central glutamatergic synapse. Science 274:972-976.

Wu GY, Cline HT (1998) Stabilization of dendritic arbor structure in vivo by CaMKII. Science 279:222-226.

Wu GY, Zou DJ, Rajan I, Cline H (1999) Dendritic dynamics in vivo change during neuronal maturation. J Neurosci 19:4472-4483. 